$14^{\text {th }}$ International Conference on

AEROSPACE SCIENCES \& AVIATION TECHNOLOGY,

ASAT - 14 - May 24 - 26, 2011, Email: asat@mtc.edu.eg

Military Technical College, Kobry Elkobbah, Cairo, Egypt

Tel: +(202) 24025292-24036138, Fax: +(202) 22621908

\title{
Discussion of Transformable Modules Spacecraft
}

\author{
Weihua Ma*, Jianping Yuan ${ }^{\dagger}$, Jianjun Luo ${ }^{\ddagger}$, Yanping $\mathrm{Bai}^{\S}$ and Shuguang $\mathrm{Li}^{* *}$
}

\begin{abstract}
As the payloads increases, the traditional spacecraft will no longer meet the future requirements from rocket carrying capacity, payloads installation and Operationally Responsive Space (ORS). Modular Spacecraft has been proposed to solve the problem. If modules have the capacity of transformation, the corresponding spacecraft, Transformable Modular Spacecraft (TMS) supposed in this paper, would be more competent for space mission. Through moving, reconnecting, transforming modules, TMS exhibits the capability of structure reconstruction and reversibility. After analyzing the transformation modes used by current spacecraft, the sliding stretch and rotating deployment are selected as ones of modular transformation mode. The plan that huge solar sail is constructed with solar panel modules as a typical TMS application is shown at last.
\end{abstract}

Keywords: Transformable Modular Spacecraft (TMS), module, transformation, sliding

\section{Introduction}

To meet the requirements from future space missions, the new payloads are needed. The traditional method is merging all the payloads on one spacecraft. Then, as the payloads increases, the traditional spacecraft would fall into conflictions, especially from rocket carrying capacity, payloads installation and Operationally Responsive Space (ORS).

- Contradiction between spacecraft volume and rocket carrying capacity. Rockets can only launch the spacecraft with limited mass and volume which decided by the engine and fairing of rockets, respectively. For tradition spacecraft, more payloads, more allowed volume requirements for fairing. It would induce a series of problems for rocket design.

- Contradiction between payloads installation and the limited working surface: payloads commonly have the special installation requirements. For example, antenna always is mounted on the selected working surface for communication. But working surface is limited; all payloads are unlikely mounted on the desired surface. It leads spacecraft adjust attitude to meet direction requirements of some payloads. So it is an unavoidable problem to coordinate installation for future more payloads.

\footnotetext{
* Ph.D. Northwestern Polytechnical University. 251 mail box, 127 Youyi west road, Xi'an, Shaanxi Province, P.R.China/whitedragon_ma@163.com

$\dagger$ Professor. Ph.D, Northwestern Polytechnical University. jyuan@nwpu.edu.cn

* Professor. Ph.D, Northwestern Polytechnical University. jiluo@ nwpu.edu.cn

$\S$ Master, Northwestern Polytechnical University.294836252@qq.com

${ }^{* *}$ Ph.D. Candidate, Northwestern Polytechnical University. lisg81@ gmail.com
} 
- Contradiction between conventional assembling and ORS. It takes long time to assemble conventional spacecraft mounted all payloads in current situation, such as communication satellite. So, conventional spacecraft will no longer quickly response to the future requirements from ORS.

To solve contradictions above, Modular Spacecraft (MS), such as F6 ${ }_{[3]}^{[1]}$, HexPak and SMARTBus ${ }^{[3]}$, put forward. By distributing the payloads into the standardize modular with some functions, not only shortens the assembling time, but also has the capability of the upgrading and extending life on orbit. By adjusting modules configurations, spacecraft presents figure fitting to rocket fairing and could avoid working surface confliction of multipayloads.

Based MS, the idea of Transformable Modular Spacecraft (TMS) is put forward to solve problem above in this paper. Besides modules reconfiguration similar to MS, the TMS still fulfill mission by module transformation.

Focusing on TMS, main works are as follows: the background of spacecraft transformation is described in Section 2. The concept of TMS and comparing between the traditional spacecraft and TMS are shown in Section 3. After analyzing the transformation modes used by current spacecraft, sliding transformation for modules is introduced in Section 4. Finally, the plan applying modular transformation in solar panel deployment to construct huge solar sail is given in Section 5.

\section{Background of Spacecraft Transformation}

In the view of figure changing, the development of transformable spacecraft can be divided into three stages: spacecraft with deployable solar panel, spacecraft with deployable payloads such as antenna and mechanical arm and the future modular spacecraft.

The original spacecraft configuration is totally fixed, without active structure, and solar cells are mounted on the body surface. Then solar panel is applied to supply the more energy required from increasing payloads. To convenient for rocket launching, solar panel are folded on ground and deployed on orbit. It can be regarded as the prime spacecraft transformation.

With the more space facilities mounted, spacecraft volume is expanded. Some facilities are folded within the faring envelopes on ground and deployed automatically in space. For instance, the future relay satellite antenna diameter is not less than 5 meters, so it must adopt the deploy way as described above ${ }^{[4]}$; Space mechanical arm is used in space operational missions widely. Space mechanical arms mounted in space shuttle and international space station consist of several arms and joints. It is folded into a small rectangle during launching, and deploys a long arm by adjusting joints in space to fulfill operations.

As the modular concept proposed, MS is paid attention to gradually. The modular design idea is that put the payloads into different function cabins and assemble these cabins to be a whole spacecraft. More assembly configuration of modules, more forms of spacecraft MS has been developed, such as Hexpak ${ }^{[2]}$, SuperBot ${ }^{[5]}$ and SmartBus ${ }^{[3]}$.

Hexpak is a scalable satellite structure consisting of stacking hexagonal modules. Scalability is supported via embedded network connectivity for pulg-in-play avionics and expansion 
bays. It as well as packs in the launch fairing, and deploys on orbit, shown in Fig.1, to solve the contradiction between the body dimension and fairing volume ${ }^{[2],[6]}$
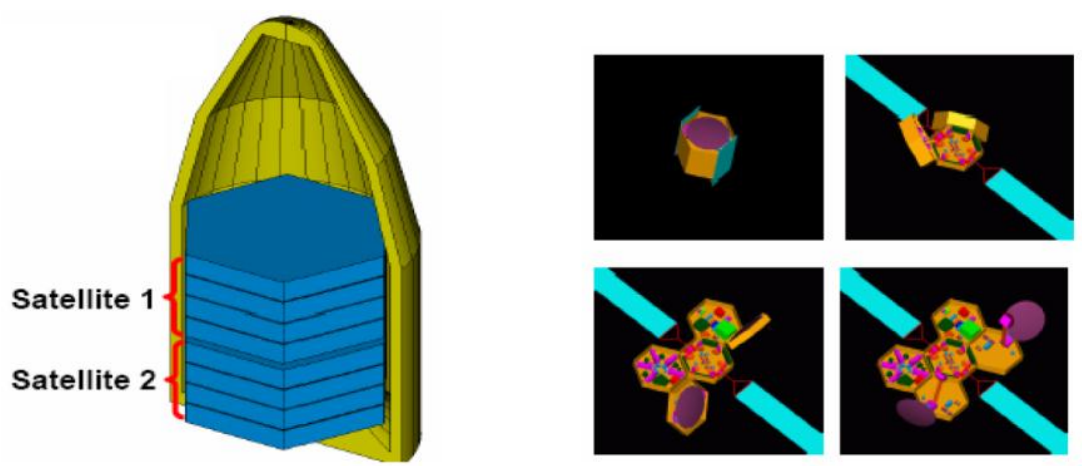

Fig. 1 HexPak Launch and Deployment Sequence

SuperBot Robotic System has been developed under the support of NASA's H\&RT program. It uses modularity and self-reconfiguration as an effective means to achieve low cost, multifunction, and adaptive capabilities. SuperBot consists of identical modes. Each module is entirely independent robot system with the abilities of energy, control, sensor, communication, and it connects with other modules by six connectors. Modules can relatively rotate to change configuration freely, shown in Fig. $2^{[5],[7]}$.
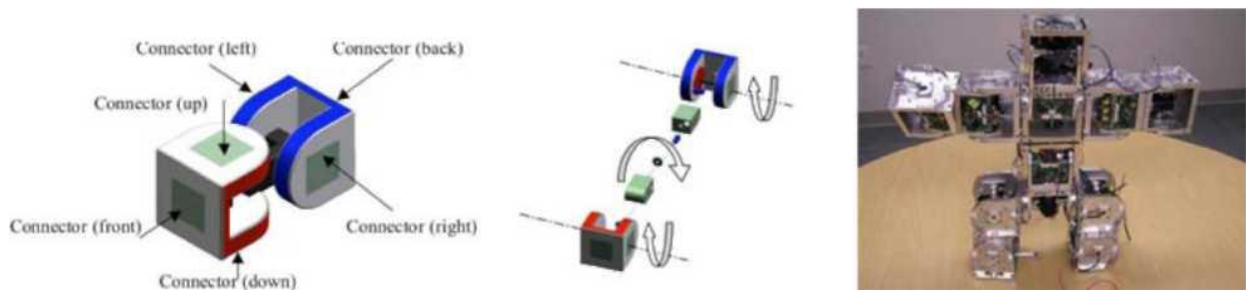

Fig. 2 SuperBot Modules and Configuration

SmartBus is developed by AeroAstro as a modular spacecraft to achieve rapidly responsive space missions. This spacecraft distributes payloads to a set of mechanical, electrical, and logical(software)standards hexagon modules, shown in Fig.3. The difference with other modular spacecraft is the way of solar panel deployment. SmartBus put three panels in standard modules. The Fold-Out Arrays module slides out, and then unfolds when it is needed $^{[3]}$.
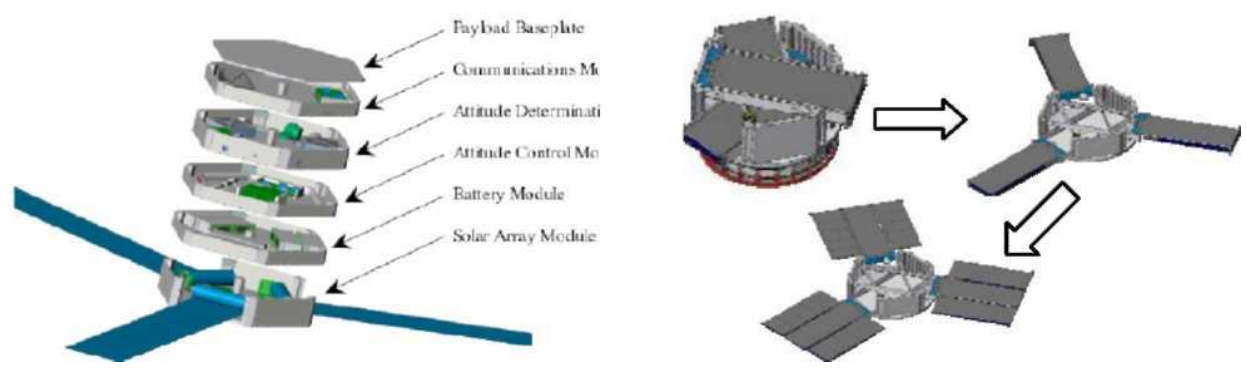

Fig. 3 A Typical SmartBus Stack 


\section{Concept and Comparing of TMS}

Based the transformation development, it is concluded that current spacecraft only presents the figure changing and the craft main mechanical structure remains unchanged during transformation. While the future transformations may occur as the case which the spacecraft's body configuration has changed so greatly that the module connects with the different structure during transformation. Therefore, it needs further analysis for the space transformation, and this just is the aim of TMS.

TMS could be termed as a system constructed by a set of standardized and reconfigurable modules connecting with universal docking interfaces. The module has active structure and self-transformation capacity. Through moving, reconnecting and transforming modules, TMS exhibits the capability of structure reconstruction and reversibility.

Structure reconstruction is that the capability of connecting with other modules by different structure during the different period, while reversibility is that spacecraft can be transformed arbitrarily within some different figure. So, TMS transformation is not only the simple unreversible changing just like solar panel which wouldn't be folded if deployed. Transformers would be the typical representation for future TMS.

Compared with traditional spacecraft and TMS, the mainly differences are as followed:

- Transformable components. Current deployable systems are usually used for flexible accessory systems (big antenna, solar panel) and the spacecraft body hasn't any change. While active components are not only the accessory systems, flexible components may be a part of the body for future transformable spacecraft. Thus, the body may be different completely after transformation.

- Driving mechanism. Current accessory systems always adopt blasting or motor to deploy. Actually, there are many means to accomplish the function of spacecraft transformation. For example, electromagnetism force utilizes repulsion and suction of magnetic field to achieve the deployment and fold. The future transformation driving facility is need to considering.

- Deployable form: Solar panel and mechanical arm always adopt rotation form to deploy currently. While the other forms, such as sliding stretching, could be adopted by transformable spacecraft.

Compared with current MS and TMS, the only difference is the modules. Current module remains unchanged during missions. While module used by TMS in future should has the ability of transformation. It means the module could be self-transforming to construct some figure required by mission while current MS have to use reconfigurable modules to realize the same requirement. The capacity of modular transformation makes TMS more competent for flexible, efficient and durable mission.

The difference of transformable components, drive mechanism, deployable form, affects not only on design of TMS, but also deployable components, force environment and dynamic modeling. So, how the modules transform must be necessary attentive technology. 


\section{Modular Transformable Mode of TMS}

There are many transformable structures. Some scholars put forward the modular connection structure for modular robot standard, shown in Fig. $4^{[8]}$. The view can be introduced to TMS. Modules transformations can be accomplished by swinging (turning), moving (sliding) and rotating the relevant components. In fact, swinging type is one of rotating type. So, such transformations could be ranged as two modes, rotating and sliding.

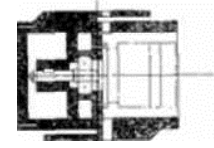

a. swinging

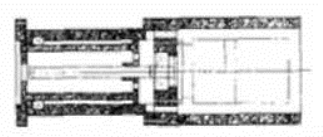

b. sliding
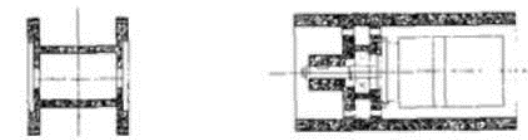

d. rotating

Fig. 4 Mechnical Modules

Although rotation gemel has been used usually in space, sliding gemel has more better kinematics performance than rotation gemel ${ }^{[9]}$. So sliding transformation is a feasible choice, too. As shown in Fig 5, component sliding can extend surface and solve the contradiction between payloads installation and the limited working surface described above.
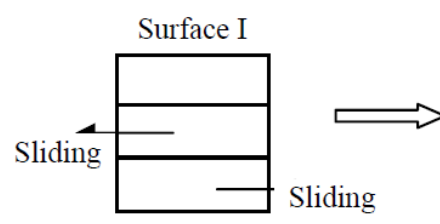
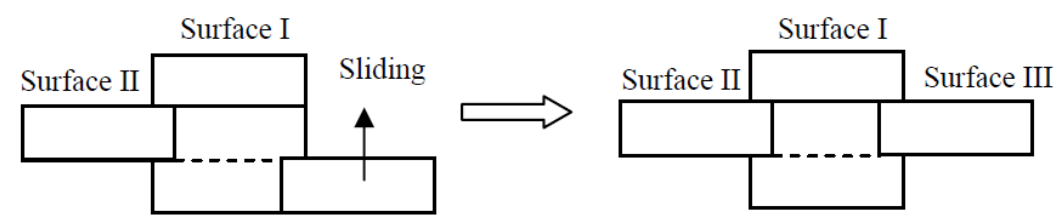

Fig. 5 Sliding Precession

\section{Solar Sail Construction based TMS}

As one of typical applications, modular transformation is implemented in a solar panel deployment plan to array the solar sail collecting space solar power. With rotating and sliding transformation, a solar panel module deploys into a new flat module. With Universal Docking Port (UDP), the flat modules connect each other to form a huge solar sail. UDP is an interface that could transfer mechanical forces and moments, electrical power, and communication.

The standard solar panel module consists of three same dimension cuboid cabins with nine solar panels all together. Cabins could be divided into two types, termed $\mathrm{Ca}$ and $\mathrm{Cb}$. Each cabin contains three solar panels. Ca cabin, centered in solar panel module, connects two $\mathrm{Cb}$ cabins by rotation gemels in its symmetrical two sides, shown in Fig.6.

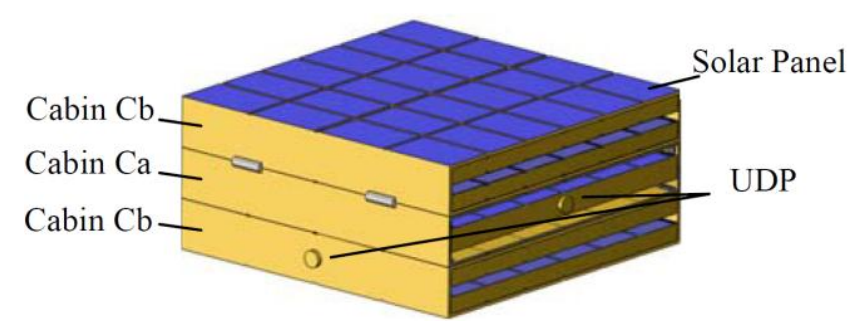

Fig. 6 Solar Panel Module 
Ca cabin consists of a nest of frames. Based sliding transformation, the smaller two embedded frames, termed as $\mathrm{Fa}$ and $\mathrm{Fb}$, could stretch in opposite direction from the cabin shell. The out surface of embedded frames is mounted a UDP. Different cabins could be connected by UDP in various space missions. On the top surface of three frames, each solar panel is mounted.
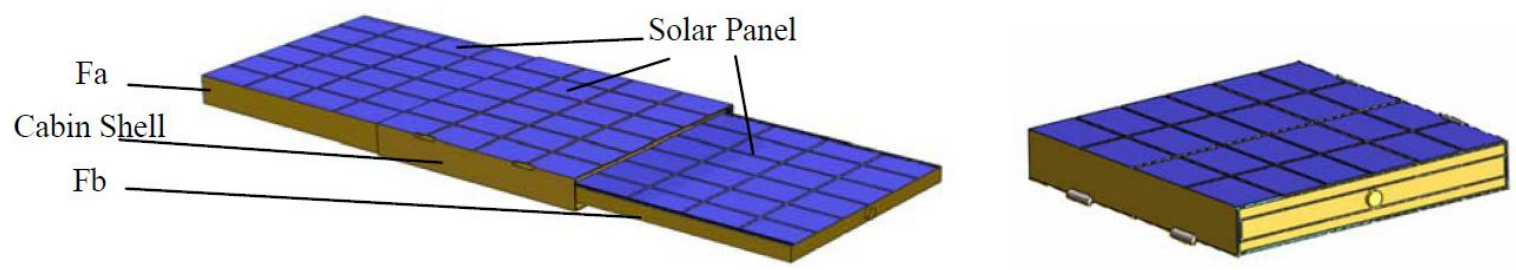

Fig. 7 Ca cabin (Unfolded and Folded)

$\mathrm{Cb}$ cabin contains a two same dimension embedded frames, termed as $\mathrm{Fc}$ and $\mathrm{Fd}$. Based sliding transformation, the two embedded frames could stretch in opposite direction from cabin, too. Two solar panels is mounted on the frames upper side and one is mounted on the upper surface of cabin. a UDP is also mounted in the side surface of cabin.
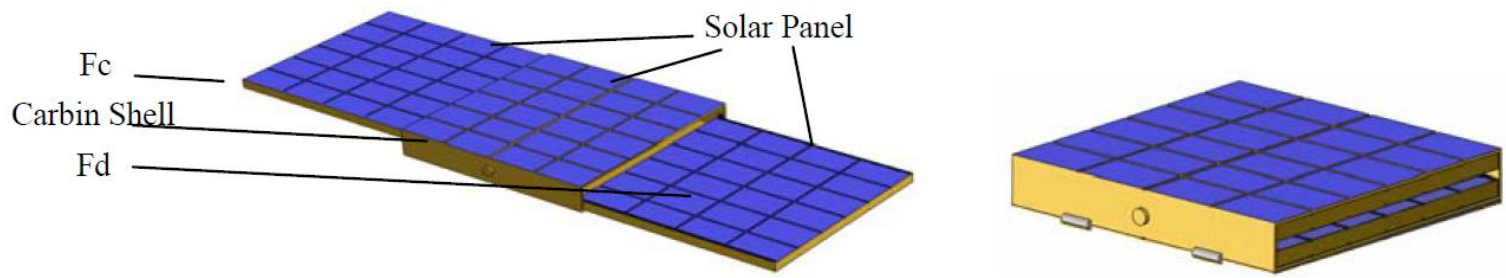

Fig. 8 Cb cabin (Unfolded and Folded)

Solar panel module cabins are folded in ground as shown in Fig. 6. And cabins are unfolded in space and module deploys into a flatter cuboid. Then embedded frames slide from cabins and the original module becomes a square flat, shown in Fig.9.
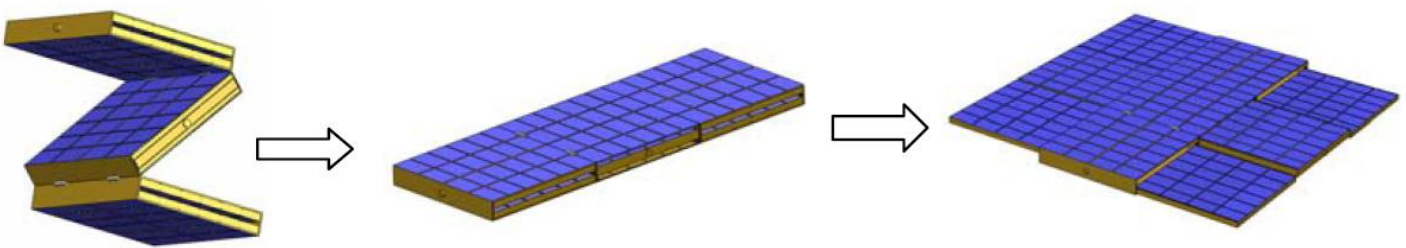

Fig. 9 Module Rotating and Sliding Deployment

The new flat could be as a new module to construct the various huge solar sail through UDPs. Solar sail presents rectangle, square, triangle and other figuration according the different configurable among the modules.
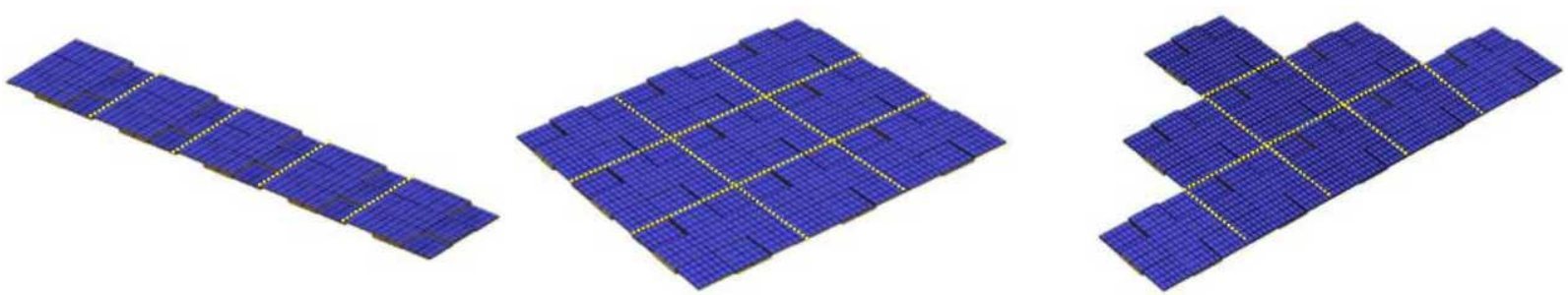

Fig. 10 Solar Sail Constructed by Modules 


\section{Conclusion}

This paper describes the TMS as a new MS with the modular self-transformation to meet the requirements from ORS, rocket carrying capacity and multiple payloads installation. Through moving, reconnecting and transforming modules, TMS exhibits the capability of structure reconstruction and reversibility for future space application. It was discussed how TMS can be used in future applications by modular sliding and rotating transformation. The design of TMS is affected by transformable components, drive mechanism, deployable form and etc. It arouses new key technologies such as modular transformation mode design, corresponding dynamic modelling, control and etc. In the future, we plan to discuss the dynamic modelling and control technology for typical modular transformation used by TMS.

\section{Acknowledgments}

This research is supported by NPU's Creativity Fund. We thank Shangjun Ma for creating the conceptual CAD model.

\section{References}

[1] Modular Space: DARPA's F6 Program. http://www.defenseindustrydaily.com/Modular- Space-DARPA-Awards-Phase-2-Systems-F6-Contract-06044, 4/27/2010.

[2] Michael Hicks, Michael Enoch, Larry Capots. HexPak Test Bed Development. $4^{\text {th }}$ Responsive Space Conference, Los Angeles, CA, 2006. id: AIAA-RS4 2006-3006. Scott A. McDermott, Luis G. Jordan. AeroAstro's SMARTBusTM: A Low-C

[3] Modular Approach Enabling Responsive Space Missions[A]. 3rd Responsive Space Conference, Los Angeles, CA, 2005, id: RS3-2005-3003

[4] Mingzhi Liu, Guifang Gao. Advances in the Study on Structure for Space Deployable Antenna. Journal of Astronautics, 2003, Vol.24, Num.1, pp.82-87. Wei-Min Shen, Mark Moll, Behnam Salemi. Mo

[5] Reconfigurable SuperBot for Space Applications. Space 2660, San Jose, California, 2006, id: AIAA 2006-7405.

[6] Steven W. Schenk and Stanley O. Kennedy, Jr. ISET Compliant Modular Multi-Mission Space Vehicle Design Feasibility for Rapid Response. 7th Responsive Space Conference, April 27-30, 20

[7] Jeremy Hsu, Superbots are the real-life Transformers http://www.msnbc.msn.com/id/31512784/ns/technology_and_science-science, 6/23/2009 Yanhui Wei, Jie Zhao, Hegao Cai. Task-based Method for Determin

[8] Reconfigurable Modular Robot. Chinese Journal of Mechanical Engineering, 2006, Vol.42, pp.93-97.

[9] Yishen Guo, Li Chen. Bi-directional Approach For Motion Planning of Space Manipulators with Prismatic Joint. Mechanics in Engineering, 2006, Vol.28, Num.1, pp.28-31. 